\title{
On the Efferent Paths of the Heart Reflex in Rabbits, Elicited by Stimulating the Carotid Sinus and Aortic Nerve.
}

\author{
By \\ Kimio Abe. \\ (安部公男) \\ (From the Physiological Laboratory of Prof. Y. Satake, \\ Tohoku Imperial University, Sendai).
}

As the heading shows, the present investigations are wholly the same in nature and scale as those reported by T.Inaba, ${ }^{1)}$ five years ago, only with a difference in species of animals; In aba experimented on dogs and cats. The methods applied here were also wholly the same as the previous, except that no anaesthesia was resorted to at all in the present experiments.

From another point of view, the present investigations may be also regarded as supplementary to a study of $\mathrm{Br}$ i c ke, ${ }^{2)}$ who was able to show about twenty years ago that the inferior cervical and stellate ganglia are playing some rôle as the efferent paths of the heart reflex in rabbits, brought about on irritating the depressor nerve. On reflecting that rather recent works show that some heart accelerating fibres are also running through the sympathetic ganglia in the thoracic cavity below the stellate ganglion, ${ }^{3)}$ the present writer attempted to conduct experiments similar to $\mathrm{Br}$ ücke, only with expanding the exclusion to the above mentioned ganglia.

\section{Methods.}

44 rabbits were used; the animal was fastened supine on the

1) T. I n a ba, Tohoku J. of Exp. Med, 1932, 18, 185.

2) Brücke, Ztschr. f. Biol,, 1917, 67, 507; Previous literature here, and H.E. Hering, Die Karotissinusreflexe auf Herz und Gefässe, Dresd. \& Leipz. 1927, p. 34, especially p. $58 \mathrm{ff}$, and Koch, Die reflektorische Selbststeuerung des Kreislaufes, Dresd. $\&$ Leipz. 1931, 63 f.

3) See: W a d a, Tohoku J. Exp. Med., 1935, 26, 546. 
table. The mean blood pressure was registered by means of a mercury manometer from the left common carotid artery or a femoral artery. In a few instances the right sided artery was used. When the blood pressure became very low during the course of experimentation, warmed Ringer fluid was infused intravenously, whereby the pressure was kept at a somewhat high level for a sufficient length of time; this happened in a few cases, such as Nos. $31 \& 34$.

The heart rate was read per ten seconds from the tracings, the respiration rate was observed by an assistant and also read from the tracings. The anal temperature was measured.

On finding the sinus nerve too delicate for cutting through and preparing the central end for applying the electrode, it was not cut, but was suspended by means of a silk thread at stimulation. The aortic nerve was prepared as usual for stimulating the central cut end.

The vagi and the median cervical and stellate ganglia were at first bilaterally laid bare with as little damage as possible. Ample time was allowed to elapse then for subsidence of effects resulting from the major operations. One sinus nerve was stimulated, followed by stimulation of the aortic nerve on the same side. Afterwards the same was repeated on the other side. Or one sinus nerve was at first stimulated and then the other; then further the aortic nerves. Induction shock at the coil distance of $8-12 \mathrm{cms}$. was usually applied, occasionally the distance was $3,5-6$ and $15 \mathrm{cms}$. The current at 13 cms. coil apart was perceptible to the tongue. The stimulation extended for 10-50 seconds.

Next the vagi were cut bilaterally, and some minutes later the stimulations were repeated with the same strength of currents. Of the median cervical and stellate ganglia the same matter was repeated.

Lastly a straight incision of skin, about $10 \mathrm{cms}$. long, was made along the fifth intercostal space, the muscle layers beneath were torn, and finally the pleura costalis was cut. The opening was rendered wide by pulling the fifth and sixth ribs apart, then the lungs were pulled over to the other side of the chest by means of the hand of the assistant, so that the lateral side of the spinal column came into sight. The thoracic sympathetic chain below the stellate ganglia, already removed, as far as that situated on the nineth rib, viz. the eighth thoracic sympathetic ganglion was isolated and nipped off by means of pincers. The operation was done on both sides. Only a small number of rabbits survived the last operation. 
Generally speaking, electrical stimulation of the carotid sinus nerve and the aortic nerve produced reduction in the arterial blood pressure, the heart and the respiratory rate. In the case of the sinus nerve however, strong stimuli often elevated the blood pressure, and particularly this occurred frequently even with moderate strength of stimuli. The rise was preceded occasionally by a transitory drop.

Data.

Effects of stimulating the carotid sinus nerves and the aortic nerves will be presented in the order mentioned.

\section{(A) The Carotid Sinus Nerve.}

(1) While the heart having the intact innervation.

On stimulating electrically the sinus nerve, the blood pressure started to fall almost simultaneously with the onset of stimulation or with latency of a few seconds, and continued to fall for ten seconds or more when a moderate strength of stimulus was applied. That level was held during the rest of stimulation and five to twenty five seconds after discontinuation of stimulation the initial level was first regained.

On stimulating the right sinus nerve, the heart rate was decreased from mean 237 beats per minute (126-294 beats) to 185 beats $(66-282$ beats), the magnitude of diminution being calculated $22 \%(0-65 \%)$ of the initial rate.

In the case of the left sinus nerve the figures were 235 beats per minute (147-306 beats), 183 beats ( $84-282$ beats) and $21 \%(0-58 \%)$. The scale of the alteration was wholly the same on both sides, and it is almost the same as that obtainable on stimulating the aortic nerve, reported by Br ücke and also by myself, as given below. (The right sinus nerve in No. 7 caused an acceleration of $7 \%$ in the pulse rate, and in three cases no variations occurred).

The above figures originate from 90 experiments, and those rê the mean arterial pressure and the respiratory rate were as follows: The pressure: the right nerve stimulation caused a fall from $104 \mathrm{mms}$. $\mathrm{Hg}$. (70-161 mms.) to 78 mms. (34-136 mms.), that is a fall of $27 \%$ (1-68\%) compared to the initial. The left nerve: From $102 \mathrm{mms}$. Hg. $(72-156 \mathrm{mms}$.) to $74 \mathrm{mms}$. (37-147 mms.), that is $28 \%(0-62 \%)$. In a few cases the pressure elevated with a following drop (On both sides in No. 3 , on the right side in Nos. $7 \& 8$ ).

The respiration rate decreased in half of the cases, while no variation took place in the other half. In a few cases an acceleration occurred. The mean 


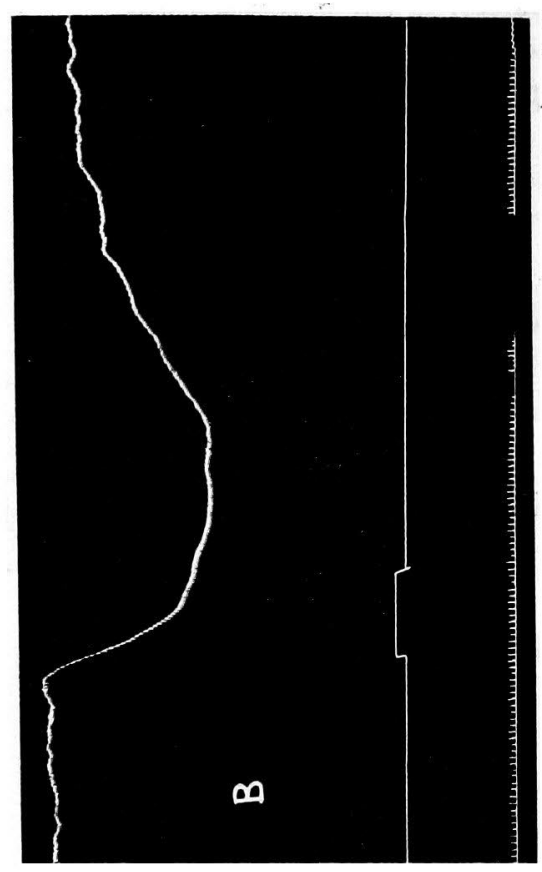

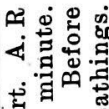

๙ึّ

on $\infty$

bo तो

$\checkmark$ 미요

땅 견. 명

ㄱㅀ ᄚ

녕

我罂

के

․ㅛ웜

을

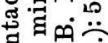

日的

秀范

ह की

.

क

ส สี้ สี

a $=$ 㷊点

की

蚛

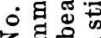

乙兄

눙ㅇㅇㅇ

ㅇ.. $=\dot{0}$

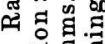

. 吉

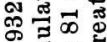

-

造䗆

. 000

웅워 의

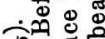
象

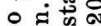

$\$$ 웜

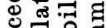

₹ 0

믄

동응

- ه 㟧

- 둥

نं $\stackrel{0}{a}$ 品

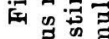

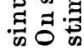

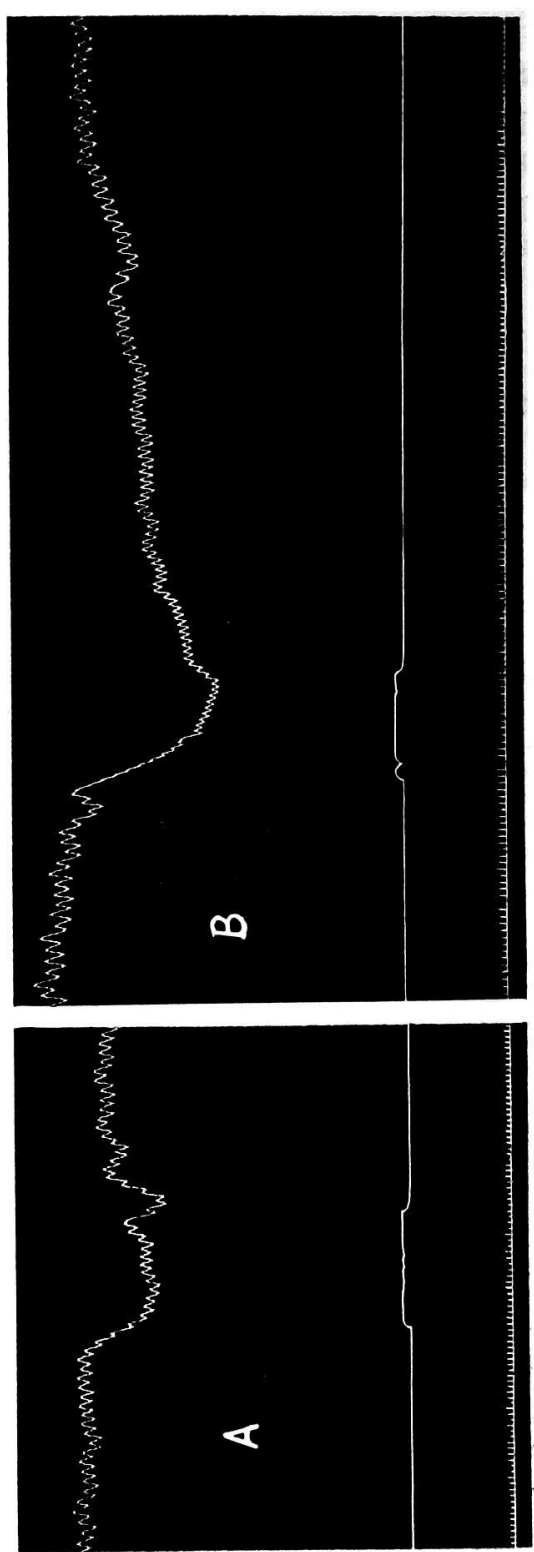

क

: ี

농ํㅇ

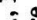

ซ் 융

घี

है

$\ddot{~}$

뭉ㅇํㅇ

훙

롤

로임

劣

क 40

융

운

๑ั

भ 웡

वं

央

๘

ี

-

的芒

.

过

a

os

品

1 00

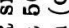

๙ क ष

¿

in

ㄴ.

응

요

๘

$\stackrel{9}{\circ} \dot{0}$

음 요

응 붕

교

언

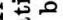

공

किष

rito

엉

Ј

स H

J 0

(1)

क्य

凷.

क

รi $₫$

ह

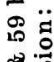



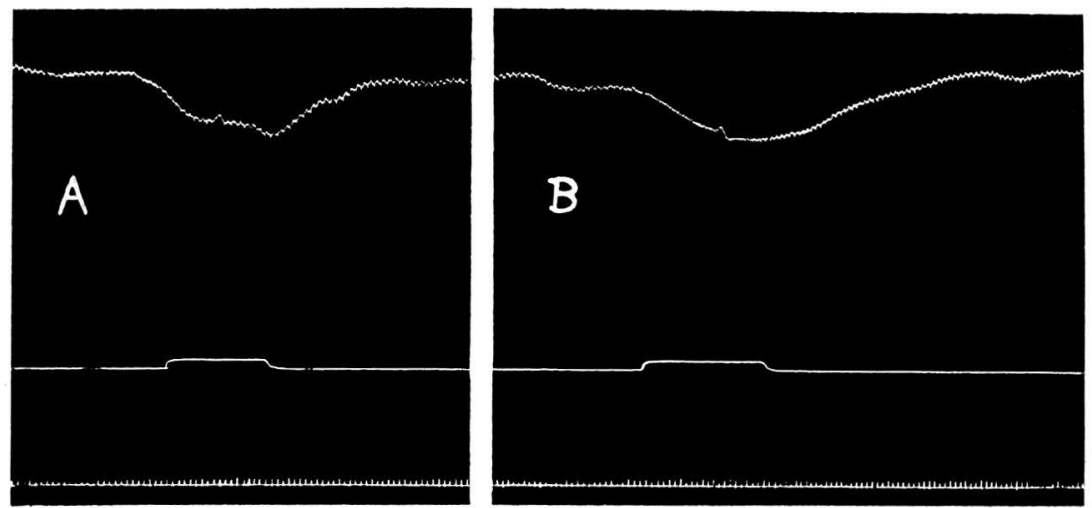

Fig. 3. (Reduced to $\frac{4}{5}$ ). After further bilateral removal of med. cerv. and stellate ganglía. A. $R$ sinus nerve stimulation. Before stimulation: $75 \mathrm{mms}$., 141 beats \& 54 breathings. On stimulation (c.d. $12 \mathrm{cms}$.) $60,132 \& 48$ respectively. B. R depressor stimulation: $74 \mathrm{mms}, 141$ beats \& 51 breathings. On stimulation (c.d. $12 \mathrm{cms}$ ): 60, 126 $\& 42$ respectively.
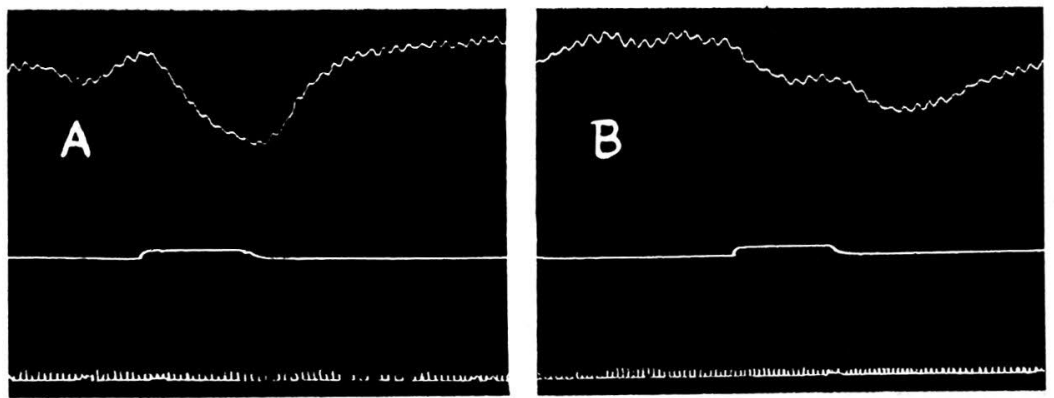

Fig. 4. (Reduced to $\frac{4}{5}$ ). After further bilateral removal of the thoracic sympathetic chains to VIII Ganglion. A. R sinus nerve stimulation. Before stimulation: $49 \mathrm{mms}$., 165 beats and 60 breathings. On stimulation (c.d. $8 \mathrm{cms}$ ) : $30,114 \& 48$ respectively. B. $R$ depressor stimulation. Before stimulation: $57 \mathrm{mms}$., 141 beats $\& 64$ breathings. On stimulation (c.d. $8 \mathrm{cms}$.): $37,138 \& 64$ respectively.

initial rate of breathing, 51 breathings per minute (42-98) was reduced to 48 breathings per minute (24-98) on stimulating a sinus nerve, 0-50\% being calculated as the ratio of the diminution against the initial frequency.

That stimulation of the carotid sinus nerve brings about as a rule a reduction in the breathing rate checks well with the previous findings of $\mathrm{Hering}$, Heymans, Koch, Moissejeff and others, while Danielopolu reported some different figures ${ }^{4}$.

4) Moissejeff, Ztschr. f. ges. exp. Med., 1926, 53, 696; Heymans and Boucka ert, J. of Physiol., 1930, 69, 254; Bouckaert and Heymans, Ibid., 1933, 79, 49; Hering, Med. Klin., 1931, 533; Koch and Mark, Ztschr. f. Kreisflauff., 1931, 23, 319; Danielopolu and Marcu, Proca, Manescu, Ztschr.f. ges. exp. Med, 1930, 70, 268. 
When the figures from case to case are examined, it may be readily noticed that in respect to the magnitude of reduction, there exists a parallelism between the blood pressure, the heart rate and the respiration, otherwise expressed, the magnitude of reductions depends upon the magnitude of the excitement of the sinus nerve mechanism.

\section{(2) After double vagotomy.}

On dividing the vagi, the blood pressure, the heart rate and the respiratory frequency remained unaltered in some cases, somewhat increased in some others and somewhat decreased in the rest. That no material alteration occurs on dividing the vagi in the pulse rate in the rabbit so long as it is fastened on the table is a well established fact.

On stimulating the carotid sinus nerve the heart rate diminished as follows: The right sinus nerve caused a reduction from the mean rate of 238 beats per minute (157-306 beats) to that of 212 beats (132$294)$, that is that of $11 \%(0-37 \%)$. The figures of the left nerve were 241 beats (168-303), 211 beats (138-294) and 11\% (1-24\%). About the same magnitude obtainable on stimulating the aortic nerve after double vagotomy, as observed by $\mathrm{Br}$ ücke and by myself, as will be presented below.

The above data were yielded on stimulating the right nerve in 30 cases al $d$ the left in 28 cases, viz. the sinus nerve in 58 cases in toto.

On stimulating the right nerve, the pulse rate was accelerated in one case (2 per cent increase) and not altered in one case, and with the left nerve an acceleration $(11 \%, 11 \% \& 1 \%)$ was recorded in three cases.

The blood pressure decreased as the mean from $107 \mathrm{mms}$. Hg. (46-156 mms.) to $90 \mathrm{mms}$. (41-153 mms.) on stimulating the right sinus nerve, and from $107 \mathrm{mms}$. (59-165 mms.) to $86 \mathrm{mms}$. (41-129 mms.) on the left nerve. The magnitude of the fall, reckoned to percentage of the initial, was on an average, both sides together, $19 \%(2-39 \%)$. In two cases of the right sided nerve a small elevation $(2 \%)$ was recorded, and in a case of the left an elevation of $26 \%$.

The respiratory rate decreased in 15 cases out of 30 right nerve stimulation, and in 7 cases out of 26 left nerve stimulation. In one case of the right side experiment and 4 cases of the other side experiment an acceleration, such as $8 \%, 20 \%, 12 \%, 11 \%$ and $14 \%$, was noted.

The present findings of the effects of stimulating the carotid sinus nerve after double vagotomy in rabbits, undoubtedly differ from those of the same kind of experiments of the previous workers, but in other species of animals, viz. dogs and cats.5) That the present data harmonize with the previous and present experience with the aortic nerve in rabbits, is as mentioned above.

5) Tournade, Hermann and Tourdan, C.r. Soc. Biol., 1929, 100, 1025; Danielopolu and $M$ arcu, Proca and Manescu, Ztschr. f. ges, exp. Med., 1930, 70, 268; Lim and H sa, Chin. J. of Physiol., 1931, 5, 40; In aba (1). 
(3) After further bilateral removal of the median and stellate ganglia.

Excision of these ganglia brought about no material changes in the figures under question.

On stimulating the right sinus nerve the pulse rate was reduced in 20 cases among $\mathbf{2 6}$, no change in 4 cases, and an increase in 2 cases ( $1 \% \& 3 \%)$, and on stimulating the left sinus nerve a reduction occurred in 21 cases out of 23 , and no alteration in the remainder. The figures show: Stimulating the right nerve reduced the pulse rate on an average from 231 beats per minute (141-297) to 213 beats (132$288)$, that is by $9 \%(0-23 \%)$, and stimulating the left from 233 beats per minute (171-288) to 194 beats (132-264), that is by $13 \%(0-40 \%)$. These figures rê the magnitude of reduction in the heart rhythm coincide well with those obtainable in the hands of the present writer on stimulating the aortic nerve, but undoubtedly larger than those of Brücke.

The arterial blood pressure fell on stimulating invariably, only in a single case where the right nerve was irritated, a slight elevation (5\%) occurred. The initial pressure level, the lowest level during the stimulation, and the ratio of the scale of fall to the initial value were respectively mean $101 \mathrm{mms}$. $\mathrm{Hg}$. (62$150 \mathrm{mms}.), 76 \mathrm{mms}$. Hg. (43-122) and $25 \%(8-48 \%)$ in the cases of stimulation of the right nerve and $102 \mathrm{mms}$. $\mathrm{Hg}$. (48-160 mms.), $81 \mathrm{mms}$. Hg. (44-145) and $20 \%(5-43 \%)$ in the cases of the left side. The magnitude of fall in the arterial pressure was therefore quite the same as that in the doubly vagotomized rabbits.

The respiratory frequency was reduced in nearly half the cases ( 24 cases ont of 49) and remained unaltered in the other half (21 cases) and in 4 cases it was accelerated $(14 \%, 25 \%, 7 \% \& 11 \%)$. The percentage of reduction was on an average $8 \%$.

(4) After further bilateral removal of the thoracic sympathetic chain to the VIII. ganglion.

This operation enfeebled considerably the general condition of the animals. In two cases Ringer fluid was intravenously administered. We were able to undertake further stimulation of the nerves only in five rabbits; the right nerve was stimulated in 4 cases, and the left in 1 case.

The heart rate diminished from the mean value of 181 beats per minute (165-198 beats) to 168 beats per minute (114-198 beats) on stimulating the right carotid sinus nerve and from 195 beats per minute to 192 beats per minute on stimulating the left nerve. On dis- 
continuing stimulating the initial rate viz. 195 beats per minute was recovered. The magnitude of diminution was calculated as $8 \%(0-$ $31 \%$ ) in the right side cases, and $2 \%$ in the left side case. It is probably difficult to catch any material difference between the scale of reduction in this set of experiments and that in the above paragraph.

In all the cases the arterial blood pressure fell on stimulation. Taking all the data together $49-90 \mathrm{mms}$. Hg., mean $73 \mathrm{mms}$. $\mathrm{Hg}$. was the level before stimulation, 31-84 mms. Hg. mean $54 \mathrm{mms}$. $\mathrm{Hg}$. was that during stimulation; therefore the scale of fall compared to the initial level was $7-38 \%$, mean $27 \%$. The scale of the fall in the blood pressure can be taken as materially similar to the cases without interfering with any nerve, or with disturbing some efferent nerves under question.

The respiratory frequency was diminished in two cases and remained unaffected in three.

\section{(B) The Aortic Nerve.}

The whole figures of the responses of the vascular system in toto upon stimulating the aortic nerve are identical to those obtainable on stimulating the carotid sinus nerve, but it seemed sometimes a little slow in the former.

\section{(1) Before cutting any nerves.}

The heart rate diminished always on exciting the aortic nerve, excepting a single case out of 46 (R. 25 cases, L. 21 ).

The heart rate before stimulation was mean 238 beats per minute (84-246), and 198 beats (84-246) on stimulating the right aortic nerve; that is a reduction of $17 \%(3-60 \%)$ resulted. That before stimulation 228 beats per minute (144-306), and 184 beats (96-282) on irritating the left aortic nerve; that is, a reduction of $20 \%(0-39 \%)$. was obtained.

The figures for both sides experiments being taken together, the blood pressure, which was $98 \mathrm{mms}$. $\mathrm{Hg}$. (65-147 mms.) was reduced to $74 \mathrm{mms}$. $\mathrm{Hg}$. $(42-118 \mathrm{mms} . \mathrm{Hg}$.) on stimulating the aortic nerve; a reduction of $25 \%$ (11$57 \%)$ ocourred.

The respiratory frequency was diminished by stimulating the aortic in 24 cases out of 46 , and accelerated in two cases. In the remainder no alteration occurred.

(2) After double vagotomy.

The pulse rate was usually reduced by stimulation of the aortic 
nerve. Only in two cases out of 38 (R. 21 cases, L. 17 cases) no variation occurred, and in a single case (L) a slight acceleration (2\%) was registered.

Stimulation of the right aortic reduced the heart rate from 237 beats per minute (186-294) to 206 beats (114-276), and that of the left from 233 beats per minute (174-291) to 201 beats (114-288 beats). The magnitude of reduction was $14 \%(0-44 \%)$ by the right aortic and $14 \%(0-45 \%)$ by the left. Both nerves exerted wholly the same magnitude of influence reflexly upon the heart rate, quite different figures from those of cats and dogs. . $^{\text {) }}$

The blood pressure fell invariably, and its magnitude was from $106 \mathrm{mms}$. Hg. (69-159 mms.) to $78 \mathrm{mms}$. Hg. (41-102 mms.) on stimulating the right aortic, from $98 \mathrm{mms}$. $\mathrm{Hg}$. (52-144 $\mathrm{mms}$.) to $68 \mathrm{mms}$. $\mathrm{Hg}$. (40-111 mms.) on stimulating the left nerve. The magnitude of fall reckoned of the initial height was $26 \%$ $(1-51 \%)$ by the right nerve and $30 \%(8-53 \%)$ by the left.

The respiratory frequency was reduced in about half the cases, viz. 20 out of 38 , and in the other $(15$ cases) no variation took place. In 3 cases where the right aortic was stimulated, an acceleration occurred (5\%,7\% \& $36 \%)$.

These results rê the heart rhythm coincide well with those yielded on stimulating the carotid sinus nerve, shown in the above chapter and those of Brücke dealing with the aortic nerve; only the slowing of the pulse rate on stimulating the aortic nerve after double vagotomy was a little smaller compared with the present, that is $8.5 \%(3-17 \%)$ against $25 \%(6-54 \%)$ before cutting the vagi. An acceleration and no variation was observed by Brücke in each one case out of 50 individuals.

(3) After further double removal of the median cervical and stellate ganglia.

On stimulating the aortic nerve a retardation occurred in 29 cases out of 34 , and an acceleration $(1 \%)$ in one case and none in 4 cases. In the cases with right nerve the initial rate of 220 beats per minute (141-303) was reduced to 202 beats (126-282), and in the cases with the left nerve they were 221 beats per minute (142-276) and 202 beats $(120-264)$ respectively. The scale of reduction in comparison to the initial rhythm was $9 \%(0-30 \%)$ and $9 \%(0-17 \%)$ respectively.

The scale of reduction on the aortic nerve stimulation was thus plainly large even after bilateral interference of vagus and the median cervival and stellate ganglia, while Brücke was not able to find any

6) Hirobata and Hasimoto, Tohoku J. of Exp. Med., 1936, 28, 231; Hasimoto and Hirohata, Ibid, 245. 
material reduction in quite the same kind of experiment. And these figures harmonize well with those in the carotid sinus experiments.

In all the experiments the arterial blood pressure fell. Before stimulating it was on an average $90 \mathrm{mms}$. $\mathrm{Hg}$. (60-134 mms.) and on stimulating $66 \mathrm{mms}$. $\mathrm{Hg}$. (41-94 mms.), mean $27 \%$ (6-46\%) being fallen.

The respiratory rate diminished in 11 out of 34 cases, underwent no alteration in 20 cases, and was accelerated in 3 cases $(7 \%, 5 \%, \& 10 \%)$. The maximum of reduction was calculated as $33 \%$.

(4) After further bilateral removal of the thoracic sympathetic chain to the VIII. ganglia.

Owing to the major operation the general condition was poor and the arterial blood pressure low. On stimulating the aortic nerve the blood pressure fell always. Before it was on an average $74 \mathrm{mms} . \mathrm{Hg}$. (57-92 mms. Hg.) and during stimulation $52 \mathrm{mms}$. $\mathrm{Hg}$. (37-86 $\mathrm{mms}$. $\mathrm{Hg}$. ), that is it was reduced by $21-40 \%$, mean $30 \%$.

The heart rate was reduced on stimulating the right aortic in 3 cases out of 4 , but in each one case of the right and left nerve no change was recorded. The initial rate and the rate during the stimulation were 141-207 beats per minute, mean 179 beats, and 138-192 beats per minute, mean 173 beats respectively, the ratio being $0-7 \%$, mean $3 \%$.

The respiratory rate was unaltered in 4 cases, and in one case of the right side stimulation it increased by $9 \%$.

Generally speaking, the scale of influence of stimulating electrically is entirely the same in the case of the carotid sinus nerve and the aortic nerve. The strength of the faradic current was taken as somewhat stronger than that perceptible by the tongue, but never so strong.

Diagrammatically speaking, the heart rate was diminished on stimulating the nerve, either carotid sinus nerve or the aortic, by twenty per cent of the initial, when all the cardiac nerves were remaining uncut, by fifteen per cent when the vagi were interfered with, by ten per cent when further the median cervical and stellate ganglia were removed, and finally by three per cent when the thoracic ganglia to the eighth ganglion were taken out. This residued reduction might be reflex inhibition of epinephrine secretion on stimulating either the sinus nerve or the aortic nerve.?

7) Heymans, C.r. Soc. Biol., 1928, 99, $1239 \& 1929,100,199 ;$ Binet and G ayet, Ibid., 1929, 100, 338; A o mura, Tohoku J. of Exp. Med., 1930, 15, 1 (Literature rê the aortic nerve here). 
The figures partly defend, as a matter of course, upon our experimental conditions; the general conditions of the animal became worse particularly after major operations, such as to remove the thoracic sympathetic ganglia.

In all the sets of experiments the blood pressure fell in almost similar scale, both in the absolute value as well as in the percentage reckoned from the initial level.

\section{(C) Stimulation of the Carotid Sints Nerve} after Cutting the Aortic Nerves.

After finishing the experiments on the aortic nerves, it was again tried to stimulate the carotid sinus nerve.

Cutting the aortic nerves left no visible alteration behind in the blood pressure level, the pulse rate, or the respiratory rate.

\section{(1) Without interfering with the cardiac nerves.}

The heart rate diminished on stimulating the right carotid sinus nerve ( 9 cases) from mean 206 beats per minute (153-246) to 152 beats per minute (120-216), and on stimulating the left sided nerve ( 9 cases) from 216 beats per minute (144-270) to 162 beats per minute (96-258 beats). The scale of reduction was calculated as $4-47 \%$, mean $25 \%$ for the right sided experiments and as $11-42 \%$, mean $26 \%$ for the left.

The blood pressure fell from $106 \mathrm{mms}$. $\mathrm{Hg}$. $(93-116 \mathrm{mms}$ ) to $74 \mathrm{mms} . \mathrm{Hg}$. (49-108 mms. $\mathrm{Hg}$.$) , that is, it fell by 7-52 \%$, mean $29 \%$ of the initial height.

The respiratory frequency diminished in 10 cases, increased in a single case and remained unaltered in 7 cases.

\section{(2) After double vagotomy.}

On stimulating the right carotid sinus nerve in 9 cases, the rate of pulse of 217 beats per minute (168-246) was reduced to 176 beats per minute (132-216), and on irritating the left nerve in 9 cases, it was reduced from 214 beats per minute $(129-264)$ to 175 beats per minute (42-228). The reduction was 4-40\%, mean $19 \%$, of the initial rate and $2-63 \%$, mean $20 \%$ respectively.

The blood pressure fell from $121 \mathrm{mms}$. Hg. (99-154 mms. Hg.) to $88 \mathrm{mms}$. Hg. ( $36-133 \mathrm{mms}$.), that is by $7-64 \%$, mean $30 \%$ of the initial.

The respiration was increased in a single case, while in the remaining it was either reduced or unaltered. 
(3) After further bilateral removing the median cervical and stellate ganglia.

18 experiments (R. 9, L. 9) were carried out.

The heart rate was 213 beats per minute (156-288 beats) before stimulating and 191 beats per minute (144-270) during stimulating the right carotid sinus nerve; and they were 215 beats per minute (136-288 beats) and 179 beats per minute (120-258) for the left side experiments. The scale of reduction was $4-17 \%$, mean $10 \%$ and $5-$ $41 \%$, mean $16 \%$ respectively, and $4-41 \%$, mean $13 \%$ in toto.

The blood pressure. fell from $114 \mathrm{mms}$. Hg. (86-127) to $83 \mathrm{mms}$. Hg. (56$125 \mathrm{mms}$. Hg.), that is by $27 \%(2-52 \%)$ of the initial level.

The respiratory frequency was reduced in half of the cases, and not influenced in the other half.

After dividing the aortic nerves, the carotid sinus nerve thus seemingly is capable of influencing more strongly the heart rate and the blood pressure, compared to the cases with intact aortic nerves. This can be rather expected from the previous reports of H. E. H ering and his co-adjutors.

\section{SUMMary.}

Rabbits were experimented on without giving any anaesthesia. They were fastened supine on the table, the arterial pressure was recorded usually from a carotid artery or a femoral.

The carotid sinus nerve was isolated from the surrounding but not cut, the aortic nerve was prepared in the cervical region and its central cut end was used for stimulation. The strength of electric current for stimulation was taken as somewhat stronger than that perceptible to the tongue. Stimulation was carried out while the heart was remaining innervated entirely intact, or the vagi cut, the median cervical and stellate ganglia were removed, and further, the thoracic sympathetic ganglia to the eighth removed.

While the blood pressure fall remained as almost the same degree under various conditions of experiments, the diminution of the heart rate was influenced by interfering with the vagi, the median cervical and stellate ganglia, and further, the middle and low thoracic sympathetic ganglia. Otherwise expressed, all these nerves convey the efferent impulse of retarding the heart beat on exciting the carotid sinus nerve and the aortic nerve. After excluding all the nerves above 
mentioned, the reflex retardation remains still, though slightly, and this might be possibly explained by an inhibition of epinephrine output causable on exciting the carotid sinus nerve and the aortic nerve.

Influence of these nerves upon the heart rate and the blood pressure is equal by both sides nerves, contrary to cats and dogs.

After the aortic nerves were interfered with, the effect of the sinus nerve became apparently enlarged. 